\title{
44. New Fractionation Method of Synaptic Vesicles in the Brain
}

\author{
By Kazuaki OHsawa and Koji Uchizono \\ Department of Physiology, Faculty of Medicine, University of Tokyo
}

(Comm. by Yasuji Katsuki, M. J. A., March 12, 1975)

Purification of synaptic vesicles from the brain homogenate is one of the most elementary procedures in neurochemistry. The principle and procedures of the isolation of synaptic vesicles seem to have been well established since the pioneering works by Whittaker ${ }^{1)}$ and De Robertis. ${ }^{2}$ However, it was suspected that the established routine methods might not always yield satisfactory results in our own preliminary experiments during the past three years, being probably due to our technical immaturity.

Being annoyed by frequent failures, we were forced to slightly modify the conventional procedures and finally could obtain synaptic vesicle fraction much purer than those by the conventional procedures with much higher success probability.

Materials and methods. $10 \%(\mathrm{w} / \mathrm{v})$ whole brain or cerebral

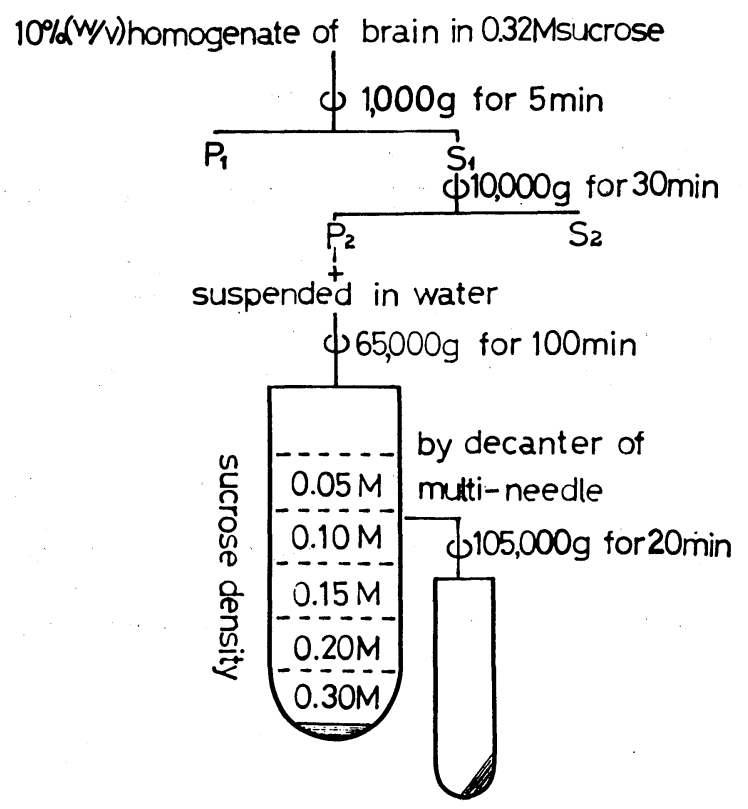

Fig. 1. Schematic illustration of the fractionation method of synaptic vesicles. 
cortex of the guinea pig was homogenized in $0.32 \mathrm{M}$ sucrose solution (adjusted at $\mathrm{pH} 7.4$ by $0.01 \mathrm{M}$ phosphate buffer) by two strokes with a teflon-glass homogenizer of $0.4 \mathrm{~mm}$ clearance. At first, the homogenate was centrifuged at $1,000 \mathrm{~g}$ for $10 \mathrm{~min}$. Then, the supernatant $\left(\mathrm{S}_{1}\right)$ was centrifuged at $11,000 \mathrm{~g}$ for $30 \mathrm{~min}$ (Fig. 1).

Fractioned membrane component of $\mathrm{P}_{2}$ precipitate was hyposmotically shocked by $\mathrm{H}_{2} \mathrm{O}$ or $0.01 \mathrm{M}$ phosphate buffer with 5 to 10 times $\mathrm{P}_{2}$ volume. This hypotonic and homogeneous whitish suspension was layered over a five-layer discontinuous sucrose gradient, containing $0.05 \mathrm{M}, 0.1 \mathrm{M}, 0.15 \mathrm{M}, 0.2 \mathrm{M}$ and $0.3 \mathrm{M}$ sucrose solution and centrifuged at $65,000 \mathrm{~g}$ for $100 \mathrm{~min}$ by a swing rotor (Hitachi RPs-25-2). A precipitate was obtained from the upper layers of each sucrose solution by the ultracentrifugation with $105,000 \mathrm{~g}$ for $20 \mathrm{~min}$. The detection of the isolated synaptic vesicles were performed both optically and electron microscopically. For optical detection, each opalescent layer which was fractioned with $65,000 \mathrm{~g}$ was checked with the Hitachi optical double beam densitometer. For electron microscopy, the pellet was double-fixed with formalin and $\mathrm{O}_{\mathrm{s}} \mathrm{O}_{4}$ and embedded in Epoxy resin. The thin sections were examined with Akashi S-500 electron microscope.
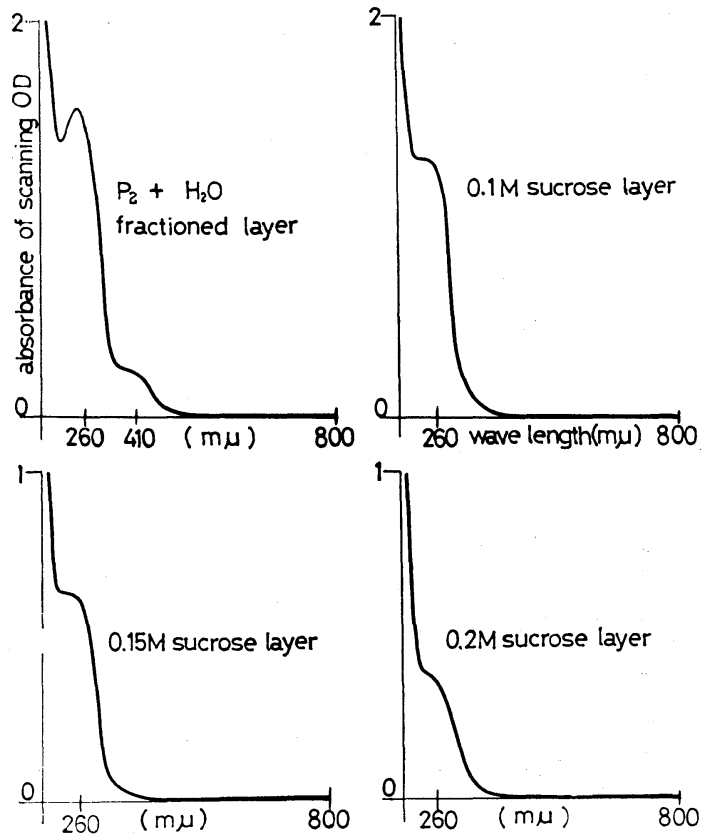

Fig. 2. Scanning optical densitographs of absorbance of wave-length from $200 \mathrm{~m} \mu$ to $800 \mathrm{~m} \mu$ for each water-shocked $\mathrm{P}_{2}, 0.1 \mathrm{M}, 0.15 \mathrm{M}$ and $0.2 \mathrm{M}$ sucrose density layer after centrifugation. 
Results. These solutions which were subjected to $65,000 \mathrm{~g}$ for 100 min centrifugation were diffuse opalescent and there was no band formation in each layer of the discontinuous sucrose gradients, although we could recognize the band when we used the hypertonic sucrose solutions of $0.30 \mathrm{M}$ or more in the previous experiments. In order to make distinction between each layer of lower density solutions, lines were drawn on the tube walls as menisci. After ultracentrifugation, each sample was sucked by a newly designed decanter ${ }^{3)}$

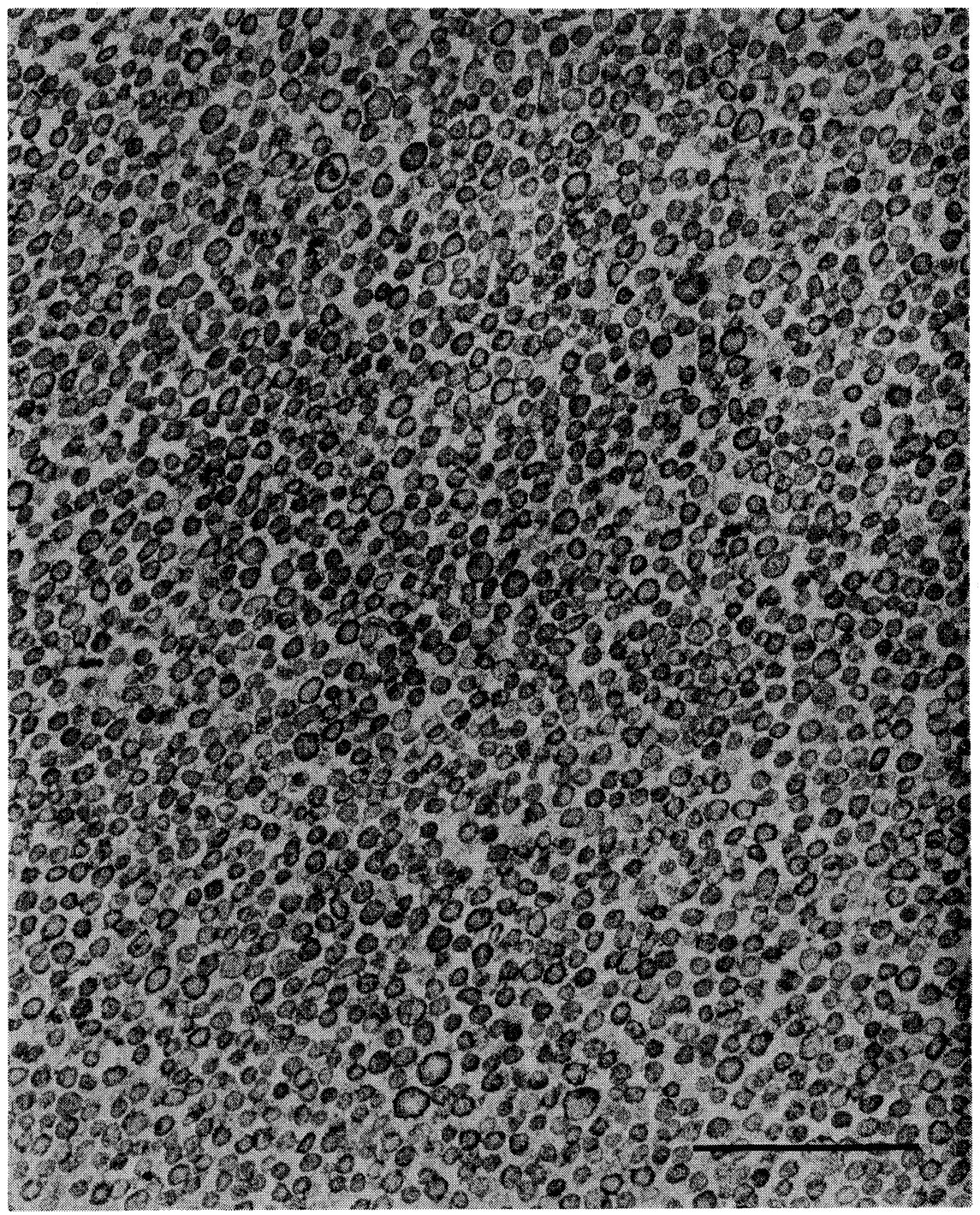

Fig. 3. Electron micrograph of synaptic vesicles obtained from the $\mathrm{P}_{2}$-pellet from $0.1 \mathrm{M}$ solution. Calibration: $0.5 \mu$. 

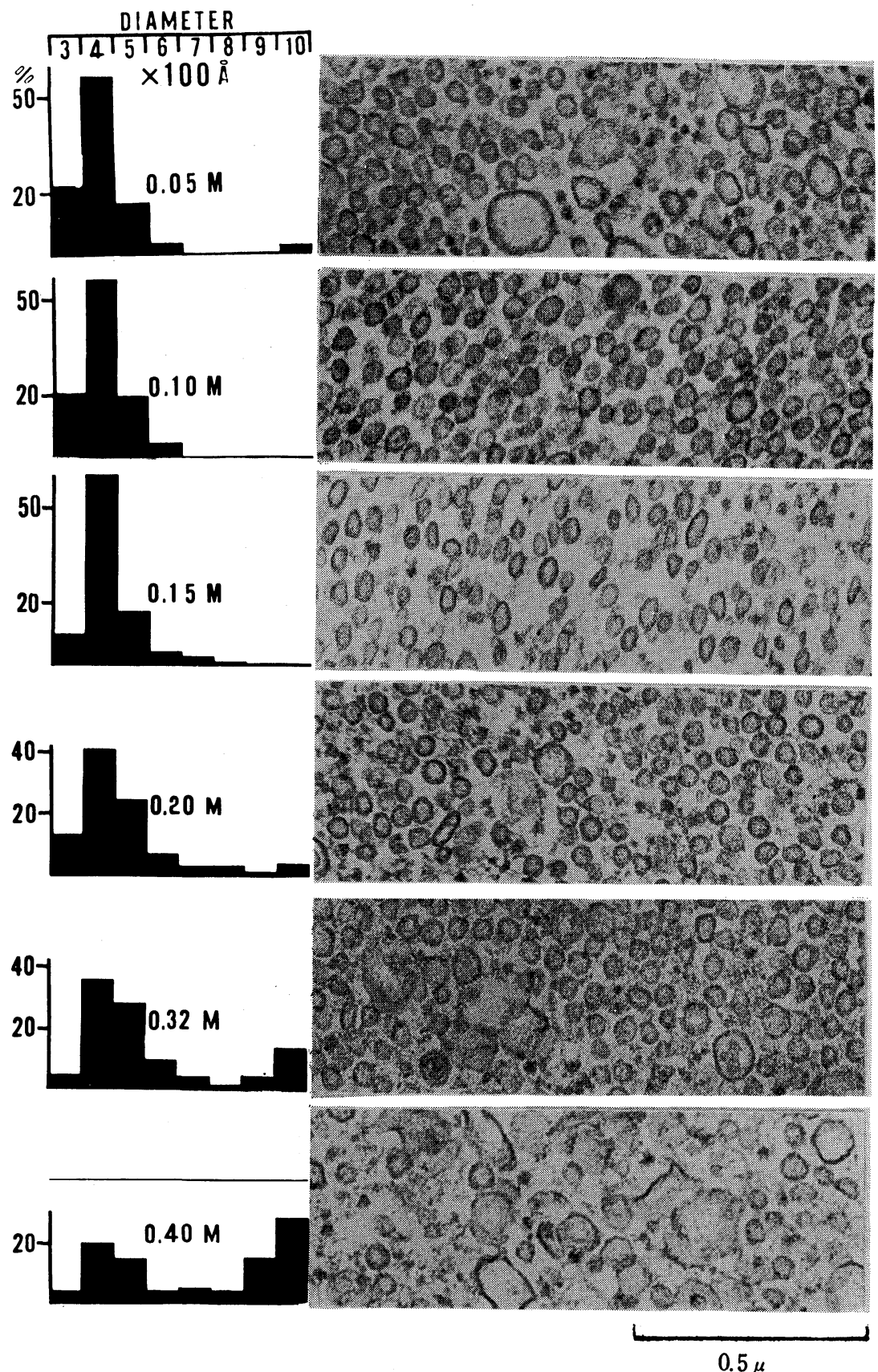

Fig. 4. Diameter distribution of synaptic vesicles and sample electron micrographs of specimens from each layer of the tube after the centrifugation. Left column shows the distribution of diameters of synaptic vesicles in each layer. See scale above. Ordinate: percentage of vesicles numbers. Abscissa: diameter of vesicles. 
from each layer of the gradients. Optical density of each fractioned layer was measured by a scanning optical densitometer (200 $\mathrm{m} \mu$ to $800 \mathrm{~m} \mu$ ) (Fig. 2).

Only a small hump was observed at $260 \mathrm{~m} \mu$ which looked similar in each sample obtained from lower sucrose density gradients from $0.1 \mathrm{M}$ to $0.2 \mathrm{M}$. Each fraction from the discontinuous sucrose density gradients was centrifuged at $105,000 \mathrm{~g}$ for $20 \mathrm{~min}$ to produce pellets as shown in Fig. 1. Routine electron microscopic observation on the pellets showed that the purest fraction of synaptic vesicles was always obtained from $0.1 \mathrm{M}$ layer of the sucrose density gradient, not from $0.32 \mathrm{M}-0.4 \mathrm{M}$ sucrose layers as usually believed to occur.

Fig. 3 shows one of electron micrographs thus obtained. Mean of vesicle diameters was $405 \pm 106 \AA$ (mean \pm SD). The second best fraction was obtained from $0.15 \mathrm{M}$ sucrose layer. $\mathrm{P}_{2}$ pellet in distilled water also contained abundant synaptic vesicles with some contamination. After ultracentrifugation, a band formation was observed in a tube of hypertonic solution more than $0.3 \mathrm{M}$ sucrose solution, but this thin band was electron microscopically proved to be overwhelmingly contaminated with organelles of more than 1,000 (Fig. 4).

Discussion. There seems to be a consensus that synaptic vesicles of brain homogenate are most effectively obtained from $0.32 \mathrm{M}$ sucrose solution by ultracentrifugation. ${ }^{1)}$ We conducted more than 200 times the same type of experiments for purification of synaptic vesicles from guinea pig brain homogenate, but we could not be as successful as we expected during the past three years. After many series of trials and errors, we finally succeeded in obtaining almost pure fraction of synaptic vesicles from $0.1 \mathrm{M}$ sucrose layer. It was revealed that very little contamination occurs in this layer. It seems that the electron micrographs of the synaptic vesicles in $\mathrm{P}_{2}$ pellet thus obtained are of the same quality as those obtained from the fixed brain tissue in vivo in perfusion experiment. It is rather curious that it contains almost no cored vesicles which we rather frequently encounter in tissue electron microscopy. The mean diameter of the vesicles is $405 \pm 106 \AA$, suggesting that these vesicles are of plain vesicles.

It must be emphasized that the tube of the ultracentrifuge looks opalescent without any band formation after the centrifugation and no recognization of a band is possible by the optical densitometry, so that it is needed to mark meniscus on the tube wall to make differentiation among various layers of different osmolarity. It is emphatically concluded that $0.1 \mathrm{M}$ sucrose solution layer is the most fruitful source of synaptic vesicles in the processes of the ultracentrifugation from 
the brain homogenate of the guinea pig. We considered that in our case the vesicles were not equilibrated permanently with $0.1 \mathrm{M}$ sucrose solution in the ultracentrifugation, however, temporarily trapped in the layer of rather hypotonic solution, because it was proved that the vesicles moved to the other layers after longer ultracentrifugation.

In summary, this new method of the synaptic vesicle isolation from brain homogenate gives much better results than the conventional methods so far reported.

Acknowledgment. All of the expenses were defrayed by the grant of the Education Ministry of Japan for Prof. K. Uchizono. The authors want to express their thanks for the help given to them by Mr. E. Kishimoto, Miss. M. Nakajima and Miss. M. Okunogi.

\section{References}

1) Whittaker, V. P.: Methods of Neurochemistry, 2, 1 (1972).

2) De Robertis, E.: J. Neurochemist., 10, 255 (1963).

3) Ohsawa, K.: in preparation (1975). 